\title{
Incidence of neuropathic pain after radiofrequency denervation of the third occipital nerve
}

This article was published in the following Dove Press journal:

Journal of Pain Research

10 April 2014

Number of times this article has been viewed

\author{
Halena M Gazelka \\ Sarah Knievel \\ William D Mauck \\ Susan M Moeschler \\ Matthew J Pingree \\ Richard H Rho \\ Tim J Lamer \\ Division of Pain Medicine, Mayo \\ Clinic, Rochester, MN, USA
}

Correspondence: Halena M Gazelka Mayo Clinic, 200 First Ave ST SW

Rochester, MN 55905, USA

Email gazelka.halena@mayo.edu

\begin{abstract}
The purpose of this study was to identify the incidence of neuropathic pain occurring after radiofrequency neurotomy of the third occipital nerve (TON). This study was conducted at a teaching hospital from January 1, 2008, to March 31, 2010. With institutional review board approval, Current Procedural Terminology codes were used to identify patients who received radiofrequency ablation (RFA) of the nerves supplying the C2-3 facet joint and the TON. The C3 dorsal ramus provides innervation to the $\mathrm{C} 2-3$ facet joint and the suboccipital cutaneous region, and procedures that included ablation to this region were reviewed for complications. Postprocedural data were collected by reviewing follow-up appointment notes and telephone calls. Included were patients who had new neuropathic pain in the distribution of the TON after RFA. They described what they were feeling as burning, tingling, or numbness. All patients who presented with complaints had normal neurologic findings and no secondary cause for their symptoms. The included patient medical records were then reviewed for severity and duration of symptoms and the need for treatment with pain medication. Sixty-four patients underwent C2-3 RFA or TON RFA, and 12 patients were identified as experiencing ablation-induced third occipital neuralgia, an incidence rate of $19 \%$. This finding suggests that patients undergoing RFA of the nerves supplying the $\mathrm{C} 2-3$ joint or TON are at risk for postprocedural third occipital neuralgia. This possibility may affect providing informed consent as well as anticipating and managing postprocedural pain.
\end{abstract}

Keywords: cervical spine, neuralgia, neurotomy, ablation

\section{Introduction}

Chronic neck pain is common in the general population, ranging in prevalence from $26 \%-71 \%{ }^{1}{ }^{1}$ Neck pain determined to be emanating from the cervical facet joints is often amenable to treatment with radiofrequency ablation (RFA) of the medial branches of the dorsal rami nerves. Pain relief from cervical RFA has been shown to last longer than 12 months in $58 \%-74 \%$ of correctly selected patients. ${ }^{2-4}$ A recent systematic review of cervical facet RFA efficacy for neck pain demonstrated a strong recommendation and moderate-quality evidence. ${ }^{5}$

Although cervical facet RFA is widely used, research on potential complications is limited. Properly performed RFA to the C2-3 facet joint necessitates neurolysis of the third occipital nerve (TON). Neuralgia can occur after this procedure. It presents as cutaneous dysesthesia in the TON's distribution. ${ }^{6}$ Sensory disturbance after cervical RFA has been documented in $2 \%-55 \%$ of patients; ${ }^{7}$ however, these numbers also include paresthesia that is not painful. Innervation of the $\mathrm{C} 2-3$ facet differs from lower cervical facet innervation (Figure 1). The deep branch of the $\mathrm{C} 3$ medial branch nerve 


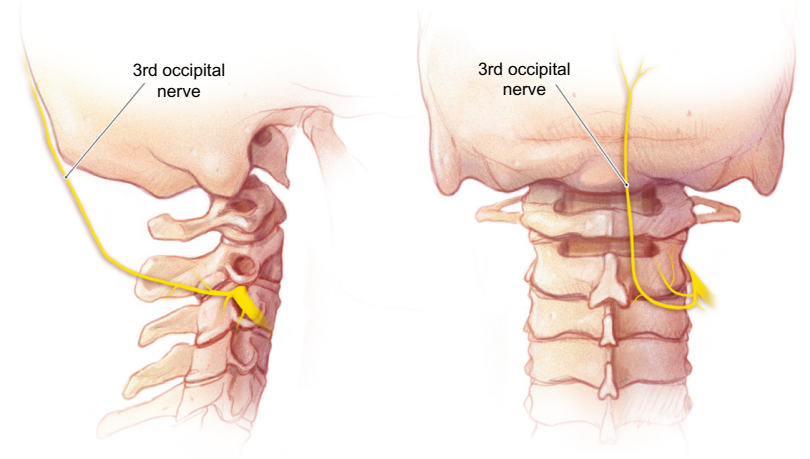

Figure I The course of the third occipital nerve as it traverses over the C2-3 facet joint.

Note: Used with permission from Mayo Foundation for Medical Education and Research @ 2011 .

provides innervation to the $\mathrm{C} 2-3$ facet, and a superficial branch of the $\mathrm{C} 3$ medial branch nerve, also called the TON, provides cutaneous innervation over the suboccipital region. ${ }^{8}$ Given the cutaneous sensory distribution, development of a painful neuralgia is a possible adverse effect after denervation procedures. This study aims to identify the incidence of neuropathic pain after RFA of the TON.

\section{Methods}

A retrospective medical record review was conducted at Mayo Clinic, Rochester, MN between January 1, 2008, and March 31, 2010. With Mayo Clinic institutional review board approval, Current Procedural Terminology codes were reviewed for all RFAs of the C2-3 facet joint or intentional RFAs of the TON. Postprocedural data including follow-up appointments and documented telephone calls were reviewed. Patients who reported new pain, described as burning, tingling, and painful numbness in the distribution of the TON, were identified, and their records were reviewed for procedure technique, the severity and duration of symptoms, and the need for pain medications.

\section{C2-3 facet radiofrequency lesioning protocol}

The radiofrequency lesioning procedure was performed with slight modifications to a previously described technique. ${ }^{8}$ The patient was placed in a prone position, and an anteroposterior view of the cervical articular pillar was obtained with fluoroscopy. Twenty-gauge, $10-\mathrm{cm}$ radiofrequency needles with 1-cm curved active tips were inserted (Baylis Medical Company, Roswell, GA, USA) in a posterior approach along a parasagittal plane, tangential to the lateral margin of the articular pillar. Keeping immediately adjacent to the lateral portion of the pillar, the needles were advanced anteriorly over the C2-3 facet joint following the convexity of the facet joint and expected location of the TON. The depth of insertion was adjusted using a lateral projection. After radiographic confirmation of the needle placement, radiofrequency electrodes were inserted through the cannulas, and impedance was verified at 300-700 ohms. Low-voltage sensory stimulation was followed by motor stimulation to confirm proper electrode placement and to exclude placement near the ventral ramus. The target nerve was anesthetized with $1 \mathrm{~mL}$ of $2 \%$ lidocaine, and conventional thermal radiofrequency lesions (Multi-RF Generator V3; Baylis Medical Company) were generated at $80^{\circ} \mathrm{C}$ for 75 seconds. Given the larger size and variation in location of the TON, a second or third lesion was created caudal and cephalad to the waist of the C2-3 joint to ensure adequate coverage. This was accomplished by using additional electrodes or by manipulating the original electrode. Placement of additional electrodes was primarily at the waist of the articular pillars of $\mathrm{C} 2$ and $\mathrm{C} 3$.

Alternatively, the originally placed electrode was further manipulated to provide additional cranial and caudal coverage of the nerve as it traverses the facet and waist of the $\mathrm{C} 3$ articular pillar. After creation of the initial lesion, some practitioners withdrew the needle slightly and made a second lesion for each needle placement to increase the overall length of lesioning along the nerve in an effort to provide a greater region of neurolysis. Figures $2-4$ present radiographic examples of needle placement.

Some patients were given corticosteroids through the radiofrequency cannula after the neurolysis before retraction of the radiofrequency probes. The type and amount of corticosteroid administered varied, but the majority of proceduralists used dexamethasone or triamcinolone.

\section{Statistical analysis}

Descriptive statistical analysis was performed, and the results are reported as absolute numbers.

\section{Results}

The medical record review identified 64 TON and C2-3 RFA procedures performed on 64 individual patients (Table 1). Overall, 12 patients identified new pain and symptoms consistent with third occipital neuralgia, yielding an incidence of $19 \%$ in these patients. The pain was described as a burning or painful dysesthesia that was new since the procedure. All patients who presented with postprocedural pain had normal neurologic findings, and there did not appear to be a secondary cause for their symptoms. 


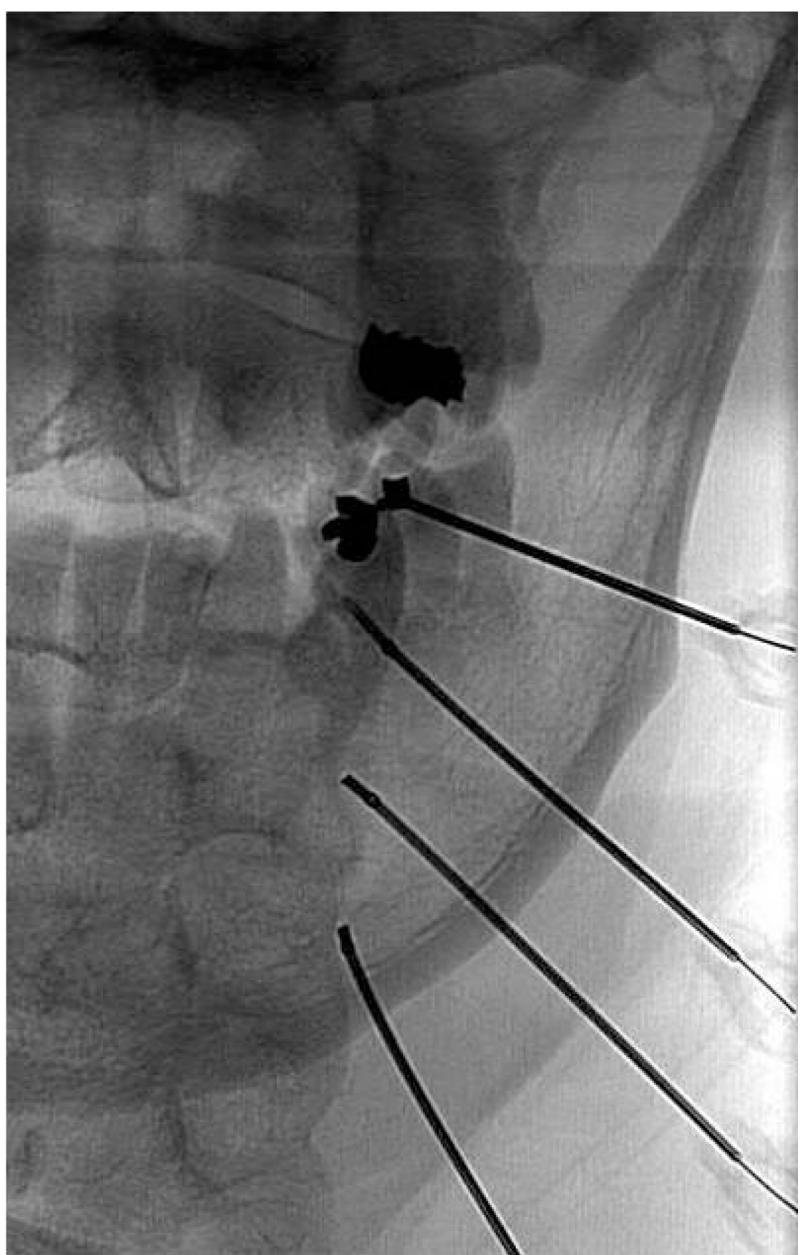

Figure 2 An anteroposterior radiograph of needle placement for third occipital nerve, C3-4, and C4-5 medial branch radiofrequency ablation.

The duration of symptoms after development of third occipital neuralgia varied greatly. Seven of the 12 patients had persistent symptoms for an average of 2.6 months after the ablation procedure (range, 1-6 months). Four of the 12 patients identified did not continue with follow-up, and the duration of symptoms remains unknown. One patient still had persistent pain at 1-year follow-up.

Ten of 12 patients (83\%) with third occipital neuralgia required treatment, which included over-the-counter analgesics, a short course of oral corticosteroids, anticonvulsants, antidepressants, and prolonged use of oral opioids. Corticosteroids were used most frequently in these patients ( 8 of 12 patients) to treat the postablation neuralgia.

\section{Discussion}

In this study, $19 \%$ of patients developed neuropathic pain in the TON distribution after fluoroscopically guided RFA of the TON and C2-3 facet when the procedure was performed according to the described technique. This incidence is less

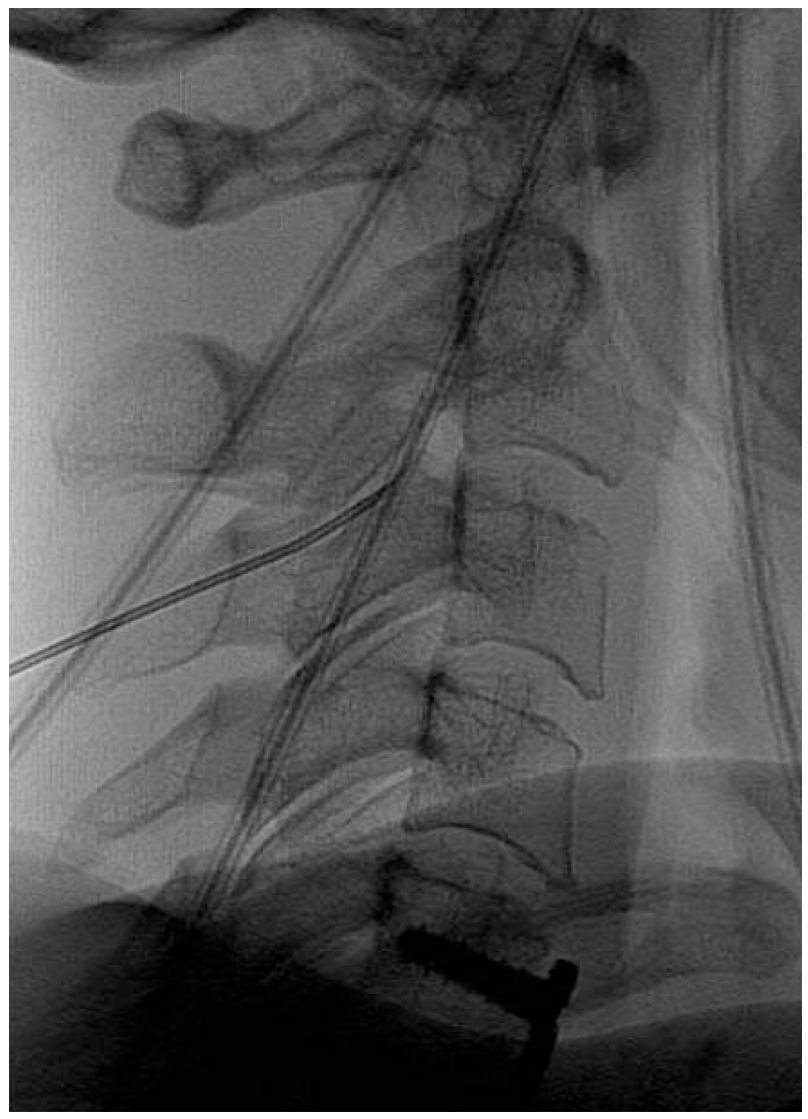

Figure $3 \mathrm{~A}$ lateral radiograph demonstrating the first needle placement for radiofrequency ablation of the third occipital nerve.

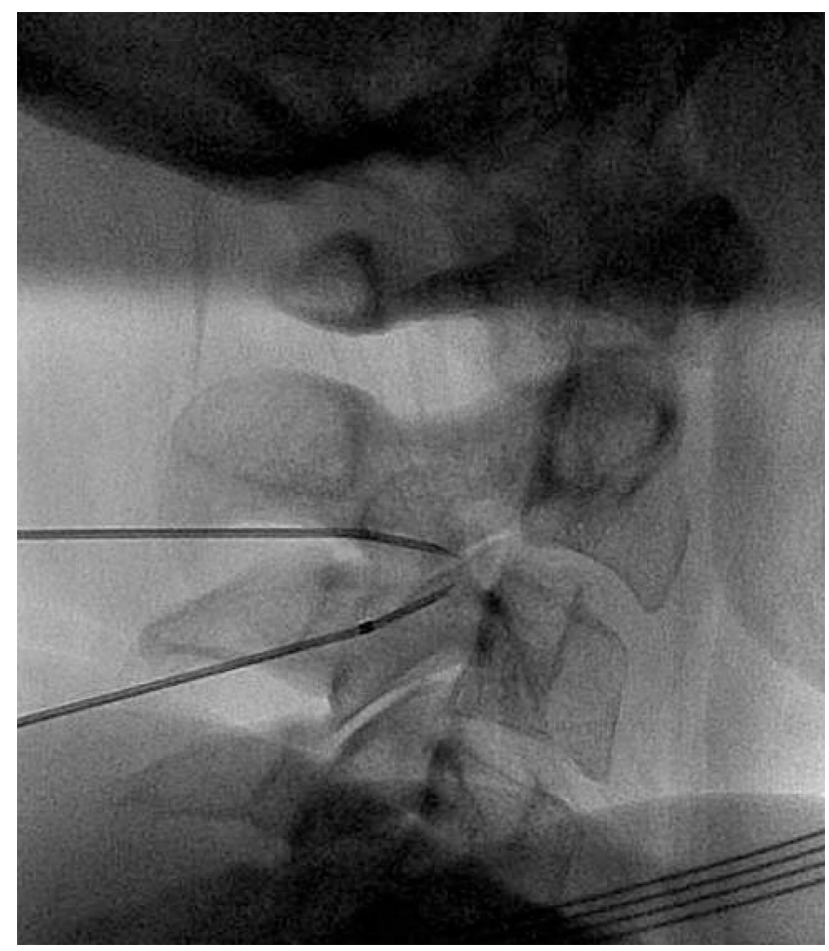

Figure 4 A lateral radiograph demonstrating the second (left) and third (right) needle placements for radiofrequency ablation of the third occipital nerve. 
Table I Yearly numbers of patients with third occipital nerve pain and the number of patients requiring treatment

\begin{tabular}{lllll}
\hline & 2008 & 2009 & 2010 & Total \\
\hline Number of procedures & 31 & 25 & 8 & 64 \\
$\begin{array}{l}\text { Number of patients with } \\
\text { postprocedural neuralgia }\end{array}$ & 4 & 7 & $\mathrm{I}$ & 12 \\
$\begin{array}{l}\text { Number (\%) of patients } \\
\text { who required treatment }\end{array}$ & $3(75)$ & $6(86)$ & $\mathrm{I}(100)$ & $10(83)$ \\
\hline
\end{tabular}

than the previously published incidence of up to $55 \% .^{7}$ Given the prevalence of chronic pain treated by RFA of the cervical spine, this is important information to discuss with patients during the informed consent process. Once informed, patients and providers together may decide to apply ablation to only the lower cervical levels, to inform patients to contact the provider should symptoms occur, or to proceed with RFA to the $\mathrm{C} 2-3$ level regardless of the risk. The majority of patients with painful neuralgia did pursue further treatment that most frequently included corticosteroids.

Previous research by Lord et $\mathrm{al}^{8}$ identified possible adverse effects of C2-3 RFA to include temporary ataxia. In contrast to our study, however, they did not find evidence for neuropathic pain in the distribution of the TON. Their study was limited to 10 patients, and they did not comment on the use of postprocedural corticosteroids. The procedural technique was consistent between the studies.

Another study, by Govind et al, ${ }^{7}$ described 49 patients who underwent TON neurotomy. Adverse effects reported in those patients included numbness, ataxia, dysesthesia, hypersensitivity, and itching. Hypersensitivity and TON dysesthesia occurred in $15 \%-55 \%$ of patients with resolution after 7-10 days, and none of the adverse effects was distressing enough to require intervention. Technical differences between that study and ours included oblique needle placement with an additional parasagittal pass.

This study has several limitations, including its retrospective identification of patients. Although most patients have clinic follow-up appointments after an intervention, many patients cancel return appointments if they are doing well. We are inferring that patients who do not return to the clinic or contact their provider after ablation have not experienced

Journal of Pain Research

\section{Publish your work in this journal}

The Journal of Pain Research is an international, peer-reviewed, open access, online journal that welcomes laboratory and clinical findings in the fields of pain research and the prevention and management of pain. Original research, reviews, symposium reports, hypothesis formation and commentaries are all considered for publication. postablation third occipital neuralgia. Also, variations in procedural techniques were not standardized. Nevertheless, this study provides a quantifiable risk of discomfort after TON RFA that likely underestimates that actual incidence of neuralgia after RFA, given that patients with minimal dysesthesia, higher pain tolerance, better coping mechanisms, and available analgesics may not report the complication. Further prospective clinical studies are needed to identify the incidence and duration of third occipital neuralgia after RFA and whether alternative treatment options such as pulsed RFA should play a greater role in treatment strategies.

\section{Acknowledgment}

This work was published as an abstract (Pain Med 2011;12[3]:510) at the 2011 annual meeting of the American Academy of Pain Medicine, Washington, DC, March 24-27, 2011.

\section{Disclosure}

The authors report no conflicts of interest in this work.

\section{References}

1. Côté P, van der Velde G, Cassidy JD, et al. Bone and Joint Decade 2000-2010 Task Force on Neck Pain and Its Associated Disorders. The burden and determinants of neck pain in workers: results of the Bone and Joint Decade 2000-2010 Task Force on Neck Pain and Its Associated Disorders. Spine (Phila Pa 1976). 2008;33(Suppl 4):S60-S74.

2. Lord SM, Barnsley L, Wallis BJ, McDonald GJ, Bogduk N. Percutaneous radio-frequency neurotomy for chronic cervical zygapophyseal-joint pain. N Engl J Med. 1996;335(23):1721-1726.

3. McDonald GJ, Lord SM, Bogduk N. Long-term follow-up of patients treated with cervical radiofrequency neurotomy for chronic neck pain. Neurosurgery. 1999;45(1):61-67; discussion 67-68.

4. Barnsley L. Percutaneous radiofrequency neurotomy for chronic neck pain: outcomes in a series of consecutive patients. Pain Med. 2005;6(4): 282-286.

5. Falco FJ, Erhart S, Wargo BW, et al. Systematic review of diagnostic utility and therapeutic effectiveness of cervical facet joint interventions. Pain Physician. 2009;12(2):323-344.

6. Boswell MV, Colson JD, Spillane WF. Therapeutic facet joint interventions in chronic spinal pain: a systematic review of effectiveness and complications. Pain Physician. 2005;8(1):101-114.

7. Govind J, King W, Bailey B, Bogduk N. Radiofrequency neurotomy for the treatment of third occipital headache. J Neurol Neurosurg Psychiatry. 2003; 74(1):88-93.

8. Lord SM, Barnsley L, Bogduk N. Percutaneous radiofrequency neurotomy in the treatment of cervical zygapophysial joint pain: a caution. Neurosurgery. 1995;36(4):732-739.

The manuscript management system is completely online and includes a very quick and fair peer-review system, which is all easy to use. Visit http://www.dovepress.com/testimonials.php to read real quotes from published authors. 